\title{
Intxaurbe, José Ramón. 2019. El empleo de signos dinámicos de adscripción religiosa en los espacios públicos y su gestión a través del instrumento jurídico del acomodo razonable. Estudio de caso de la comunidad Sij. Pamplona: Thomson Reuters Aranzadi, 524 p.
}

doi: http://dx.doi.org/10.18543/djhr.1902

El estudio que se recoge en esta monografía tiene como objetivo mostrar la eficacia de la técnica del acomodo razonable, nacida en el mundo anglosajón, en la gestión de la diversidad. El autor circunscribe la aplicación de esta técnica al ámbito del empleo de signos dinámicos, prefiero la calificación de personales, de adscripción religiosa en los espacios públicos.

Utiliza un método comparativo diacrónico que describe el origen de la figura del acomodo razonable en Norteamérica y sincrónico en el que el objeto de la comparación es, de un lado, el Derecho antidiscriminatorio y en concreto la aplicación de la técnica del acomodo razonable en un ámbito como el uso de los signos personales en sistemas jurídicos anglosajones, en concreto, Canadá y Estados Unidos, y, de otro, el Derecho antidiscriminatorio en el ámbito europeo y español, en concreto, el test de proporcionalidad aplicado por los tribunales europeos y españoles con la finalidad de determinar semejanzas, diferencias y mostrar que el acomodo razonable supone un paso más en la tutela antidiscriminatoria que el test de proporcionalidad y proponer su incorporación al ordenamiento jurídico español.

Así pues, los instrumentos jurídicos que se comparan exigen, por otro lado, una aproximación tópica, que parte de los casos concretos, de la argumentación y decisiones adoptadas por los órganos jurisprudenciales en uno y otro sistema jurídico. En el caso del sistema jurídico continental no se descuida la importancia de las normas, algo que muestra una rigurosa utilización del método comparado. El trabajo se estructura en cinco capítulos. Los capítulos primero y segundo se centran en el derecho español abordando el marco normativo que sirve de soporte a la utilización de signos religiosos personales como manifestación del derecho fundamental de libertad religiosa. Asimismo, se apoya en una completa jurisprudencia que le permite mostrar los criterios elaborados en la resolución de los conflictos que se han ido planteando en los distintos ámbitos, dedicando un apartado al ámbito educativo y laboral, así como a otros supuestos. En el análisis de la jurisprudencia tiene en cuenta no solo la jurisprudencia española, sino también la jurisprudencia del Tribunal Europeo de Derechos Humanos (en adelante TEDH) y de 
la Comisión de Derechos Humanos de Naciones Unidas (en adelante CDHNU). Pone de relieve, muy acertadamente, las limitaciones que la aplicación del margen de apreciación tiene en las sentencias del TEDH a la hora de establecer estándares mínimos de protección y garantías de esta manifestación de la libertad religiosa. El peso que se da a los ordenamientos internos de los Estados miembros del Consejo de Europa dificulta la creación de un estándar mínimo común. En consecuencia, sus pronunciamientos no pueden ser trasladados de forma automática a todos los Estados sino que deben pasar por el filtro del ordenamiento interno de cada uno de ellos.

Es interesante la comparación de estas decisiones del TEDH con las de la CDHNU. Los supuestos de hecho que se comparan son semejantes y se desarrollan en Francia. La laicidad tal y como es entendida por este país ha sido un elemento clave para que el TEDH, aplicando el margen de apreciación, resolviera a favor de Francia, mientras que la CDHNU, apoyándose en el test de necesidad y proporcionalidad, rechaza la pretensión de la parte demandada, Francia, y decide a favor de la libertad religiosa del recurrente. Pone el énfasis en el análisis de fondo y no meramente formal de la protección de la libertad religiosa considerando que no se ha demostrado que se ponga en riesgo la neutralidad, ni el perjuicio a los derechos de los demás. No se puede concluir, se dice, que con la expulsión de la educadora se haya contribuido a mitigar presión o proselitismo alguno sobre la comunidad de menores y sus progenitores, ni que se haya evitado la estigmatización de una comunidad religiosa (§§ 8.7, 8.8, 8.9). En cambio, se concluye, se advierte una discriminación indirecta que vulnera el art. 26 del Pacto Internacional de Derechos Civiles y Políticos.

En otras ocasiones, el TEDH ha aplicado el test de necesidad en un análisis de fondo del caso exigiendo al demandado, Polonia, la prueba de que no había otra alternativa menos lesiva al derecho fundamental (caso Jakóbski c. Polonia). En el derecho español el test de proporcionalidad ha sido utilizado en algunas sentencias, su aplicación ha llevado a amparar la libertad religiosa.

Para el autor no es suficiente el test de proporcionalidad dado que su interpretación y aplicación por la jurisprudencia no garantiza, cuando no se realiza un análisis de fondo, la protección de las manifestaciones religiosas, en concreto los símbolos religiosos y, especialmente, en el ámbito laboral lleva a la adopción de medidas muy radicales como el despido. Para paliar este resultado plantea el autor la propuesta de que la negación del acomodo cuando este sea posible sea considerada una causa de discriminación. Hay que recordar, así lo hace el autor, que la Directiva europea 2000/78/CE, de 27 de noviembre relativa al 
establecimiento de un marco general para la igualdad de trato en el empleo y la ocupación, y su transposición por la Ley 62/2003, de 30 de diciembre de medidas fiscales, administrativas y sociales, recoge la figura del ajuste razonable que sería el equivalente al acomodo razonable, los elementos que la integran son los mismos. Sin embargo, la circunscribe a la discapacidad. Bastaría, pues, con extenderla a los supuestos de libertad religiosa.

El capítulo tercero se dedica a mostrar la aplicación del acomodo razonable en Norteamérica, implementado en el ámbito de las relaciones laborales. El resultado de este análisis indica las limitaciones de su aplicación en Estados Unidos. En la ponderación de los intereses en juego entre la libertad de empresa y la libertad religiosa, la valoración y el peso de la carga excesiva acaba primando la libertad de empresa en detrimento de la libertad religiosa. En la experiencia canadiense, en cambio, se advierte "un esfuerzo por avanzar hacia una concepción de la igualdad en clave sustantiva y no asimilacionista. (...) intentando agotar las posibilidades de negociación, sin menoscabo de la relación laboral». Los casos analizados le permiten concluir que "la obligación de acomodar podría imponerse como excepción a lo previsto en un convenio colectivo (...) cuando no existan mejores alternativas disponibles o cuando su puesta en práctica no interfiera con el desarrollo del negocio y sin que el acomodo pueda suponer la pérdida de un puesto de trabajo o relegación de una persona que actualmente está ocupando un determinado puesto de trabajo". El cuarto capítulo se dedica al estudio en profundidad de las dificultades con las que una comunidad, la sij, se encuentra por portar signos de identidad religiosos en diversos ámbitos, escolar, laboral, prisiones y otros. Las resoluciones judiciales en Norteamérica le permiten profundizar en la figura del acomodo razonable, como una manifestación de la igualdad material y no de una mera tolerancia. Destaca de forma especial la aportación de Canadá al dotar de contenido un concepto jurídico indeterminado como el de carga excesiva, desgranando los aspectos argumentativos relevantes como «la autonomía de la voluntad de la persona religiosa»; "la razonabilidad como elemento de la proporcionalidad» que se va a medir con argumentos de fondo y no meramente especulativos o hipotéticos; así como la función dialogante que acompaña a esta figura y que otorga un papel relevante a la negociación, distinguiéndose, así, de otras figuras que también contribuyen a la flexibilización del ordenamiento como la exención de la regla general, útiles para el reconocimiento de la diversidad. La articulación de la figura del acomodo razonable en el marco de la discriminación indirecta le permite establecer su conexión con el test de 
necesidad y proporcionalidad, criterio de argumentación arraigado en nuestra tradición jurídica. Establece, así, elementos comunes a ambas tradiciones que la facilitarán en el quinto y último capítulo la propuesta con la que termina su estudio.

En el quinto y último capítulo, el autor muestra las semejanzas del Derecho antidiscriminatorio europeo con el norteamericano, centrándose en la discriminación indirecta. La jurisprudencia española, tal como señala el autor, proporciona cada vez con más frecuencia casos en que el juez aplica el test de proporcionalidad para decidir acerca de conflictos en que el uso de símbolos personales es la causa. No siempre se resuelve a favor de la libertad religiosa, pero en alguna ocasión se pone de relieve la proporcionalidad de la medida que limita el ejercicio de este derecho. No obstante, el autor sostiene que la extensión del acomodo razonable, se recuerda que ya existe el equivalente ajuste razonable limitado a la discapacidad, a la libertad religiosa supondría una mayor garantía para este derecho. Esta propuesta, no parte de cero, cuenta con una base argumentativa semejante al acomodo utilizada por la jurisprudencia europea y nacional como el test de proporcionalidad con los elementos que le integran (juicio de idoneidad, juicio de necesidad y juicio de proporcionalidad en sentido estricto). También tiene un apoyo normativo, la figura del ajuste razonable, que le proporciona la Directiva europea 2000/78/ $\mathrm{CE}$, de 27 de noviembre relativa al establecimiento de un marco general para la igualdad de trato en el empleo y la ocupación, y su transposición por la Ley 62/2003, de 30 de diciembre de medidas fiscales, administrativas y sociales. La propuesta se limitaría, pues, a la extensión de la figura del ajuste razonable a aquellos supuestos en que la causa sea la libertad religiosa. Propuesta que es apoyada por un sector de la doctrina española no desdeñable. Si bien la extensión del ajuste razonable a la libertad religiosa supondría una base normativa que reforzaría la tutela antidiscriminatoria no evitaría los riesgos de una interpretación meramente formal. La experiencia de Estados Unidos es un ejemplo de como una interpretación formal de los elementos del acomodo razonable con el límite de la carga excesiva conduce a primar los intereses empresariales en detrimento de la libertad religiosa. No veo claro que se imponga de forma obligatoria y se prevean sanciones si no se aplica esta fórmula. La interpretación de los elementos que integran la figura permite al juez un ámbito de discrecionalidad necesario para apreciar caso por caso la solución más razonable y proporcionada. No siempre la pretensión de quienes alegan la protección de la libertad religiosa es razonable, no lo es cuando pone en juego los derechos de los demás. 
No quiero terminar sin realizar una valoración muy positiva de este estudio; estamos ante un excelente trabajo, que muestra la madurez y el rigor científico del autor. Aborda todos los ámbitos de interés. No huye de los problemas, por el contrario, los aborda, argumenta bien y hace inteligentes propuestas de solución. Su lectura es obligada para quien quiera conocer el estado de la cuestión de esta materia.

Adoración Castro Jover Universidad del País Vasco/Euskal Herriko Unibertsitatea 


\section{Copyright}

Deusto Journal of Human Rights / Revista Deusto de Derechos Humanos is an Open Access journal; which means that it is free for full and immediate access, reading, search, download, distribution, and reuse in any medium only for non-commercial purposes and in accordance with any applicable copyright legislation, without prior permission from the copyright holder (University of Deusto) or the author; provided the original work and publication source are properly cited (Issue number, year, pages and DOI if applicable) and any changes to the original are clearly indicated. Any other use of its content in any medium or format, now known or developed in the future, requires prior written permission of the copyright holder.

\section{Derechos de autoría}

Deusto Journal of Human Rights / Revista Deusto de Derechos Humanos es una revista de Acceso Abierto; lo que significa que es de libre acceso en su integridad inmediatamente después de la publicación de cada número. Se permite su lectura, la búsqueda, descarga, distribución y reutilización en cualquier tipo de soporte sólo para fines no comerciales y según lo previsto por la ley; sin la previa autorización de la Editorial (Universidad de Deusto) o la persona autora, siempre que la obra original sea debidamente citada (número, año, páginas y DOI si procede) y cualquier cambio en el original esté claramente indicado. Cualquier otro uso de su contenido en cualquier medio o formato, ahora conocido o desarrollado en el futuro, requiere el permiso previo por escrito de la persona titular de los derechos de autoría. 